\section{BETALAÍNAS, COMPUESTOS FENÓLICOS Y ACTIVIDAD ANTIOXIDANTE EN PITAYA DE MAYO (Stenocereus griseus $\mathrm{H}$.)}

\section{BETALAINS, PHENOLIC COMPOUNDS AND ANTIOXIDANT ACTIVITY IN PITAYA DE MAYO (Stenocereus griseus $\mathrm{H}$.)}

\author{
Leticia García-Cruz ${ }^{1}$, Yolanda Salinas-Moreno ${ }^{2 \star}$ \\ y Salvador Valle-Guadarrama
}

${ }^{1}$ Investigaciones Forestales, Agricloas, Fisiología Poscosecha, Departamento de Ingeniería Agroindustrial, Universidad Autónoma Chapingo. 56230, Chapingo, Estado de México. ${ }^{2}$ Laboratorio de Calidad de Maíz, Instituto Nacional de Investigación Forestal Agrícola y Pecuarias. Apartado Postal 10. 56230, Chapingo, Estado de México.

${ }^{*}$ Autor para correspondencia (yolasm@gmail.com)

\section{RESUMEN}

La pitaya de mayo (Stenocereus griseus $\mathrm{H}$.) es una cactácea columnar que se encuentra en zonas áridas y semiáridas de México. Sus frutos son jugosos y de coloraciones que van desde naranja hasta el púrpura. El color del fruto se debe a las betalaínas, que son compuestos nitrogenados hidrosolubles. En este estudio se analizó el fruto de dos variedades de pitaya: roja $(\mathrm{PR})$ y naranja $(\mathrm{PN})$, en cuanto al contenido de betalaínas totales (betacianinas + betaxantinas), fenoles solubles totales y ácidos fenólicos, así como el poder antioxidante que se evaluó mediante el ensayo DPPH y cálculo del $\mathrm{IC}_{50}$. En PR se obtuvo un contenido de $347.3 \pm$ $21.0 \mathrm{mg}$ de betalaínas totales (BET)/100 g muestra seca, mientras que en la PN se encontraron $215.0 \pm 36.2 \mathrm{mg}$ de BET/100 g de muestra seca. El contenido de fenoles solubles totales fue de $166.5 \pm 14.4$ y $52.8 \pm 3.8$ mg de equivalentes de ácido gálico (EAG)/100 g de pulpa seca, para los frutos de PR y PN, respectivamente. De los ácidos fenólicos (AF), los libres se encontraron en mayor proporción que los glucosilados y esterificados. La PN tuvo mayor contenido de AF que la PR. La PR mostró un valor de $\mathrm{IC}_{50}$, que es la concentración del extracto con la cual se logra una reducción de $50 \%$ del radical $\mathrm{DPPH}$, de $59.8 \pm 0.32 \mu \mathrm{M}$, en tanto que en la $\mathrm{PN}$ fue de $161.7 \pm 4.8 \mu \mathrm{M}$, lo que muestra una mayor capacidad antioxidante en la PR. La actividad antioxidante de los frutos de pitaya se atribuye principalmente a la presencia de betalaínas puesto que los fenoles se encuentran en menor proporción que ellas. Los frutos de $S$. griseus representan una alternativa para incrementar y diversificar la ingesta de antioxidantes entre la población de las zonas áridas y semiáridas de México.

Palabras clave: Stenocereus griseus, ácidos fenólicos, actividad antioxidante, DPPH, betacianinas, betaxantinas.

\section{SUMMARY}

Stenocereus griseus H. (pitaya de mayo) is a columnar cactus that can be found growing in the arid and semi-arid lands of México. It produces juicy fruits with colored flesh ranging from orange to red-purple. The color of the fruit is due to the presence of betalains, which are water soluble nitrogen-containing compounds. In this study we analyzed fruits of two varieties of $S$. griseus $\mathrm{H}$.: red (PR) and orange (PN), by measuring their composition in total betalains (betacyanins + betaxanthins), total soluble phenolics, and phenolic acids. The antioxidant activity of the fruits was also determined by DPPH assay and $\mathrm{IC}_{50}$. Total betalains content of red pitaya (PR) was $347.3 \pm 21.0 \mathrm{mg}$ of total betalains (BET)/100 g of dry flesh (DF), while in orange pitaya (PN) it was $215.04 \pm 36.16 \mathrm{mg}$ of BET/100 $\mathrm{g}$ DF. Total soluble phenolics (FST) content in the fruits of PR was $166.5 \pm 14.4$, and in PN was of $52.8 \pm 3.8$ mg equivalents of gallic acid (EAG)/100 g DF. Free phenolic acids (AFL) were found in a higher proportion than those glucosylated and esterified acids. The PN had higher content of AFL than the PR. IC ${ }_{50}$, which represents the concentration of the extract needed to reduce $50 \%$ of the $\mathrm{DPPH}$, for the fruits of $S$. griseus was $59.8 \pm 0.32 \mu \mathrm{M}$ for PR, and 161.7 $\pm 4.8 \mu \mathrm{M}$ for $\mathrm{PN}$, thus showing a higher antioxidant activity (AA) for $\mathrm{PR}$. The AA in S. griseus is attributed to betalains rather than to phenolics which were present in a lower proportion. S. griseus represents an alternative to increase and diversify the consumption of antioxidants in the population of arid and semiarid lands of México.

Index words: Stenocereus griseus, antioxidant activity, DPPH, betacianins, betaxantins, phenolics acids.

\section{INTRODUCCIÓN}

Son innegables los beneficios que acarrea a la salud humana el mantener una dieta elevada en frutas y verduras, beneficios que se atribuyen principalmente al poder antioxidante de los fitoquímicos contenidos en estos alimentos, entre los cuales destacan los compuestos fenólicos (Shahidi 2009) ampliamente distribuidos en el reino vegetal. Las betalaínas también son fitoquímicos considerados como potentes antioxidantes (Cai et al., 2003), sin embargo su presencia está restringida a solamente algunas familias de plantas relacionadas con el orden Caryophyllales, dentro de la cual destacan los géneros Beta, Amaranthus, Opuntia e Hylocereus (Cai et al., 2003; Stintzing et al., 2005). En los últimos años han proliferado los estudios sobre las betalaínas y sus propiedades en varias especies de los géneros Opuntia (Sreekantha et al., 2007; Osorio-Esquivel et al., 2011) e Hylocereus (Wu et al., 2006; Herbach et al., 2007). El género Stenocereus ha sido menos estudiado, no obstante que se caracteriza, al igual que Opuntia e Hylocereus, por producir frutos jugosos con pulpa de atractivos colores.

La pitaya de mayo (Stenocereus griseus $\mathrm{H}$.) es una cactácea columnar originaria de México. Sus frutos son bayas poliespermáticas de forma globosa u ovoide, con espinas caducas; la pulpa puede ser de color anaranjado, rojo o púrpura. El color característico de sus frutos se debe a las betalaínas, pigmentos naturales hidrosolubles con nitrógeno en su estructura que se sintetizan a partir del aminoácido tirosina. Las betalaínas se dividen en dos grupos: betacianinas, que brindan tonalidades rojas y se forman por condensación de una estructura ciclo-DOPA (dihidroxifenilalanina) con el ácido betalámico, y betaxantinas que proporcionan coloraciones amarillas y se sintetizan a partir de diferentes compuestos amino y el ácido betalámico (Strack et al., 2003; Gandía-Herrero et al., 2010).

Además de dar coloración a los frutos que las contienen y poseer actividad antioxidante, las betalaínas son reconocidas por otras importantes actividades biológicas, tales 
como la inducción de la quinona reductasa, potente enzima de detoxificación en la quimio prevención del cáncer (Azeredo, 2009), y su actividad antiproliferativa de células de melanoma maligno (Wu et al., 2006).

Los frutos que contienen betalaínas también poseen fenoles de diferentes tipos, excepto antocianinas, pues estas dos clases de pigmentos son mutuamente excluyentes $(\mathrm{Wu}$ et al., 2006). En algunas especies el contenido de fenoles es mayor que el de betalaínas, y en otras es lo contrario. En pitahaya (Hylocereus spp.) Wu et al. (2006) reportaron un contenido mayor de fenoles solubles totales $(42.4 \mathrm{mg}$ equivalentes de ácido gálico (EAG)/100 g de pulpa fresca) que de betalaínas ( $10.3 \mathrm{mg}$ equivalentes de betanina / $100 \mathrm{~g}$ de pulpa fresca); en betabel (Beta vulgaris L.), Kujala et al. (2000) señalaron un contenido mayor de betalaínas respecto a FST. En varias especies del género Opuntia los valores de FST fueron mayores que los de betalaínas (Cayupán et al., 2011).

Las investigaciones realizadas en pitaya de mayo se han enfocado principalmente en la composición proximal y características físicas de los frutos (Luna-Morales y Aguirre, 2001; Luna-Morales, 2006). Sin embargo, también es importante estudiar los rasgos funcionales de estos frutos, para impulsar su desarrollo como fuente de compuestos antioxidantes. Por ello, los objetivos de este trabajo fueron determinar el contenido de betalaínas y compuestos fenólicos, así como la actividad antioxidante de la pulpa de dos variedades de pitaya de mayo.

\section{MATERIALES Y MÉTODOS}

\section{Material vegetal}

Los frutos que se analizaron fueron de dos variedades de pitaya; roja y naranja, seis de cada una, fueron colectados en huertos familiares del municipio de Tepexi de Rodríguez, Puebla, cuando estaban en estado de madurez comercial, en mayo de 2011.

\section{Extracción y cuantificación de betalaínas}

De cada fruto se tomó una muestra de $2 \mathrm{~g}$ de pulpa fresca macerada y se colocó en un matraz Erlenmeyer al que se añadieron $20 \mathrm{~mL}$ de metanol acuoso $80 \%(\mathrm{v} / \mathrm{v})$. La mezcla se sonicó por $10 \mathrm{~min}$ (Bath sonicador, Branson model 2510®; Danbury, USA), previo a su agitación por $20 \mathrm{~min}$ en un agitador horizontal model G10® (New Jersey, USA) a temperatura ambiente y en oscuridad. Cada muestra se centrifugó a $2200 \mathrm{x}$ g por $10 \mathrm{~min}$ en una centrífuga Hettich zentrifugen ${ }^{\circledR}$ Mod. Universal 32 (Germany). El sobrenadante se guardó y el residuo se sometió a una segunda extracción con la metodología descrita. Los sobrenadantes se juntaron y se filtraron con papel Whatman Núm. 4, para luego concentrarlos a sequedad en un rotavapor Buchi ${ }^{\circledR}$ Mod R-215 (Switzerland), a $40{ }^{\circ} \mathrm{C}$. Las muestras de la variedad roja fueron re-suspendidas en $10 \mathrm{~mL}$ de metanol acuoso a $80 \%(\mathrm{v} / \mathrm{v})$ y en $5 \mathrm{~mL}$ para la variedad naranja, y luego almacenadas en viales color ámbar bajo condiciones de congelación hasta su posterior análisis.

El contenido de betacianinas y betaxantinas se cuantificó según lo descrito por Castellanos-Santiago y Yahia (2008), mediante la absorbancia de los extractos de betalainas a 538 y $483 \mathrm{~nm}$ en un espectrofotómetro Perkin Elmer Lambda $25 \circledR$ UV/Vis (USA). Para la conversión de las unidades de absorbancia en unidades de concentración se utilizó la expresión: $B(m g / g)=(A x F D x P M x V) /(\varepsilon x P x L)$, dónde $B$ es betacianinas o betaxantinas, $A$ es la absorbancia a 538 $\mathrm{nm}$ para betacianinas y $483 \mathrm{~nm}$ para betaxantinas, $F D$ es el factor de dilución al momento de leer en el espectrofotómetro, $P M$ es el peso molecular (Betanina $=550 \mathrm{~g} / \mathrm{mol} \mathrm{e}$ Indicaxantina $=308 \mathrm{~g} / \mathrm{mol}), V$ es el volumen del extracto, $\varepsilon$ es el coeficiente de extinción molar (Betanina $=60000 \mathrm{~L} /$ mol.cm, e Indicaxantina $=48000 \mathrm{~L} / \mathrm{mol} . \mathrm{cm}$ ) y $L$ es la longitud de la celda $(1 \mathrm{~cm})$.

\section{Determinación de fenoles solubles totales (FST)}

Esta determinación se hizo a partir del extracto metanólico obtenido para la cuantificación de betalaínas, mediante el método de Folín-Ciocalteu (Singleton y Rossi, 1965). A $100 \mu \mathrm{L}$ del extracto se añadieron $125 \mu \mathrm{L}$ del reactivo FolinCiocalteu $1 \mathrm{~N}$ (dilución 1:2 del reactivo comercial $2 \mathrm{~N}$ ), y se agitó y dejó reaccionar por $6 \mathrm{~min}$, para luego neutralizar la reacción con la adición de $1250 \mu \mathrm{L}$ de carbonato de sodio $\left(\mathrm{Na}_{2} \mathrm{CO}_{3}\right)$ a $19 \%(\mathrm{p} / \mathrm{v})$; luego se aforó a $3 \mathrm{~mL}$ con agua destilada, se agitó y se dejó reposar a temperatura de cuarto en oscuridad por $90 \mathrm{~min}$. Las muestras se centrifugaron a $15300 \mathrm{x}$ g por 10 min para eliminar la turbidez. La absorbancia se midió con un espectrofotómetro Perkin Elmer Lambda $25 \circledR$ UV/Vis (USA) a $760 \mathrm{~nm}$. Se elaboró una curva patrón de ácido gálico para expresar el contenido de FST en función de este ácido.

\section{Cuantificación de ácidos fenólicos}

Los ácidos fenólicos presentes en el extracto metanólico se aislaron con la metodología descrita por Bakan et al. (2003). En forma breve, se pesaron $0.2 \mathrm{~g}$ de muestra seca y se hicieron dos extracciones con $10 \mathrm{~mL}$ de metanol a $80 \%$, se concentró parcialmente a presión y temperaturas reducidas para eliminar parte del metanol. El concentrado se llevó nuevamente a un volumen de $20 \mathrm{~mL}$ con agua destilada. Para obtener los ácidos fenólicos libres (AFL), el pH de la solución se ajustó a 2 y se hicieron dos extracciones líquido-líquido con $20 \mathrm{~mL}$ de acetato de etilo en 
cada extracción. La fase orgánica se concentró a sequedad en un rotavapor Buchi ${ }^{\circledR}$ Mod R-215 (Switzerland), el residuo se re-suspendió en $1 \mathrm{~mL}$ de metanol grado HPLC y se almacenó a $-4{ }^{\circ} \mathrm{C}$ hasta su posterior análisis. La fase acuosa se aforó a $20 \mathrm{~mL}$ con agua destilada y dividió en dos partes iguales; una se trató con $10 \mathrm{~mL}$ de $\mathrm{HCl} 2 \mathrm{~N}$ y se almacenó a $4{ }^{\circ} \mathrm{C}$ durante $1 \mathrm{~h}$, para de ahí obtener los ácidos fenólicos glucosilados (AFG); la otra parte se trató con $10 \mathrm{~mL}$ de $\mathrm{NaOH} 2 \mathrm{~N}$ y se almacenó en oscuridad por $3 \mathrm{~h}$, para luego extraer los ácidos fenólicos esterificados (AFE). Posteriormente se ajustó el pH a 2 en ambas fracciones y se siguió la misma metodología que en el caso de los AFL. La cuantificación de ácidos fenólicos totales de cada una de las fracciones se determinó con el método de Folín-Ciocalteu descrito anteriormente.

\section{Actividad antioxidante}

La capacidad antioxidante de las betalaínas presentes en las muestras de pitaya roja y naranja se determinó utilizando una solución $60 \mu \mathrm{M}$ de DPPH disuelto en metanol a 80 $\%$, con el método reportado por Cai et al. (2003) que utiliza el radical estable 2,2-difenil-1-picrilhidrazil (DPPH) (Sigma Aldrich Co). Para desarrollar la reacción en una celda se colocaron $200 \mu \mathrm{L}$ del extracto metanólico de betalaínas y se agregaron $2800 \mu \mathrm{L}$ de la solución de DPPH, y se midió la absorbancia a $515 \mathrm{~nm}$, en un espectrofotómetro Perkin Elmer Lambda $25 \circledR$ UV/Vis (USA). Inicialmente la lectura se tomó cada minuto hasta el minuto cinco, posteriormente cada 5 min hasta completar 2 h, que según Cai et al. (2003) es el tiempo necesario para que la reacción se estabilice. La inhibición o reducción del radical libre DPPH se midió en porcentaje (\% DPPH) mediante la ecuación: \% DPPH = [ $\left.\left(A_{0}-A_{e}\right) / A_{0} x 100\right]$, donde $A_{0}$ es la absorbancia de la muestra al tiempo cero y Ae es la absorbancia de la muestra examinada en cada uno de los tiempos (Liu y Yao, 2007). La absorbancia al tiempo cero $\left(A_{0}\right)$ fue la obtenida al mezclar $200 \mu \mathrm{L}$ de metanol a $80 \%$ con $2800 \mu \mathrm{L}$ de la solución $60 \mu \mathrm{M}$ de DPPH.

Con el fin de determinar el $\mathrm{IC}_{50}$, el cual indica la concentración a la que se tiene $50 \%$ de reducción del DPPH, se prepararon diferentes concentraciones del extracto y se les midió el porcentaje de DPPH reducido al final de un perio- do de incubación de $2 \mathrm{~h}$. Los datos obtenidos de porcentaje de reducción de DPPH se graficaron con respecto a la concentración y se calculó la concentración a la cual se alcanzó 50 \% de reducción de DPPH, mediante una regresión logarítmica de tres parámetros. Al valor de concentración obtenido se le consideró como $\mathrm{IC}_{50}$.

\section{Análisis estadístico de datos}

Se utilizó un diseño experimental completamente al azar, y con los valores obtenidos para las diferentes variables se hizo un análisis de varianza (ANOVA) y comparación de medias por el método de Tukey $(\alpha=0.05)$ con el paquete estadístico SAS (SAS Institute, 2002). Todos los análisis se hicieron por triplicado, excepto la actividad antioxidante que se realizó por duplicado.

\section{RESULTADOS Y DISCUSIÓN}

\section{Cuantificación de betalaínas}

El contenido de betalaínas totales (betacianinas + betaxantinas) en la pulpa de la pitaya roja (PR) fue mayor ( $\mathrm{P} \leq$ $0.05)$ que en la pulpa de la pitaya naranja (PN) (Cuadro 1). En la PR predominaron las betacianinas (BC) en tanto que en la PN fueron las betaxantinas (BX). Las BC se asocian con coloraciones rojo-violeta mientras que en las BX se asocian con coloraciones amarillas, por lo que la proporción de BC y BX observada en los frutos de los dos ecotipos de pitaya aquí analizados es acorde con el color de su pulpa. Estos valores de betalaínas en frutos de pitaya son parecidos a los informados por Castellanos-Santiago y Yahia (2008) para varias especies del género Opuntia, pero superiores a los de pitahaya roja (H. polyrhizus) reportados por Wu et al. (2006) de $10.3 \pm 0.22 \mathrm{mg} / 100 \mathrm{~g}$ de pulpa fresca, y que los de Wybraniec y Mizrahi (2002) quienes señalaron una variación de 23 a 39 mg equivalentes de betanina/100 g de pulpa fresca en ocho variedades e híbridos de Hylocereus, género en el que no se ha reportado presencia de betaxantinas.

\section{Cuantificación de fenoles solubles totales $\mathrm{y}$ ácidos fenólicos}

En el extracto de pulpa el contenido de fenoles solubles

Cuadro 1. Contenido (en $\mathrm{mg} / 100 \mathrm{~g}$ de muestra seca) de betalaínas totales (BET), betacianinas (BC) y betaxantinas (BX) en dos muestras de pitaya de mayo (Stenocereus griseus $\mathrm{H}$.).

\begin{tabular}{lccc}
\hline Muestra & BET & BC & BX \\
\hline Pitaya roja & $347.30 \mathrm{a} \pm 20.98$ & $199.6 \mathrm{a} \pm 24.32$ & $147.61 \mathrm{~b} \pm 18.21$ \\
Pitaya naranja & $215.04 \mathrm{~b} \pm 36.16$ & $37.6 \mathrm{~b} \pm 7.54$ & $177.37 \mathrm{a} \pm 30.18$ \\
\hline
\end{tabular}

Medias con letras iguales en una columna, no son estadísticamente diferentes (Tukey, 0.05). 
totales (FST) de PR resultó considerablemente mayor que en PN (Cuadro 2). En pitahaya (H. polyrhizus) se han reportado valores de FST de $42.4 \pm 0.04 \mathrm{mg}$ de EAG /100 g de muestra fresca (Wu et al., 2006), que son similares a los de la pitaya roja aquí analizada, si se considera que el contenido está expresado en muestra seca. No obstante, son menores a los $15.5 \mathrm{mg}$ EAG /g de muestra seca registrados en betabel por Kujala et al. (2000). En frutos de garambullo (Myrtillocactus), Guzmán-Maldonado et al. (2010) encontraron valores que variaron de $9.12 \pm 1.28$ a $10.46 \pm 0.48 \mathrm{~g}$ $\mathrm{EAG} / \mathrm{kg}$ de muestra fresca, que también son más elevados que los observados en los frutos de pitaya analizados.

Dado que el ensayo de Folin-Ciocalteau no es específico para fenoles, pues compuestos como azúcares y algunos aminoácidos también lo reducen (Robbins, 2003), los valores de FST en el extracto metánolico de los frutos de pitaya podrían estar sobreestimados. Entre los fenoles solubles en el extracto metanólico de la pulpa de pitaya están los ácidos fenólicos, que pueden encontrarse en forma libre o conjugados mediante enlaces éter (glucosilados) o éster (esterificados) a azúcares u otros ácidos. El contenido de ácidos fenólicos fue mayor en la PN que en la PR (Cuadro 2). En Cactáceas como el garambullo se ha reportado la presencia de los ácidos gálico y caféico (Guzmán-Maldonado et al., 2010), mientras que en betabel se menciona un éster del ácido ferúlico (Kujala et al., 2000).

\section{Actividad antioxidante}

El poder antioxidante de un extracto o fruto se puede expresar en función del porcentaje de DPPH reducido (Villanueva-Tiburcio et al., 2010). Al respecto, si no se considera la concentración de antioxidantes en el extracto o fruto es poco confiable hacer comparaciones entre extractos o frutos diferentes. Para evitar esta situación se recomienda estimar la capacidad antioxidante con el $\mathrm{IC}_{50}$ (Cai et al., 2003), que representa la concentración del extracto con la cual se obtiene $50 \%$ de reducción del DPPH presente en la reacción. Mientras menor sea el valor de $\mathrm{IC}_{50}$ mayor será la ca- pacidad antioxidante.

En este trabajo se encontró que a medida que aumenta la concentración de betalaínas en los extractos de pitaya, el porcentaje de DPPH reducido se incrementa (Figura 1). En pitaya roja una concentración de $135.07 \mu \mathrm{M}$ reduce 76 $\%$ del radical DPPH, en tanto que en la pitaya naranja se requiere una concentración de $307.01 \mu \mathrm{M}$ para lograr una reducción de 67 \%. Estos resultados indican que los metabolitos presentes en los dos extractos de pitaya son diferentes, y pueden relacionarse con el tipo de betalaínas. Se ha informado que las betacianinas son antioxidantes más potentes que las betaxantinas (Azeredo, 2009), y estas últimas son las predominantes en la pitaya naranja. Los valores obtenidos de porcentaje de DPPH reducido se encuentran por arriba de lo reportado en frutos de frambuesa(Rubus idaeus) en diferentes estados de madurez, los cuales oscilaron de 50 a 70 \% (Peña-Varela et al., 2006), aunque en esta especie los metabolitos responsables de la actividad antioxidante son las antocianinas. El $\mathrm{IC}_{50}$ de la pitaya naranja fue de $161.7 \pm$ $4.8 \mu \mathrm{M}$, que es más del doble que el de la roja (59.8 \pm 0.32 $\mu \mathrm{M})$, lo que significa que el poder antioxidante de la pitaya roja es mayor que el de la naranja. Sin embargo, el poder antioxidante de $S$. griseus $H$. también puede atribuirse a los compuestos fenólicos presentes en sus frutos, y aunque están en menor proporción que las betalaínas se consideran potentes antioxidantes (Torres-Rodríguez et al., 2011).

\section{CONCLUSIONES}

El contenido de betalaínas en la pitaya de mayo ( $S$. griseus) es diferente entre los dos colores de fruto analizados, pues las betacianinas son las más abundantes en la pitaya roja y las betaxantinas en la pitaya naranja. Dado que las betalaínas se encontraron en mayor proporción que los fenoles, se consideran estos compuestos como los que más contribuyen a la actividad antioxidante observada en los frutos de $S$. griseus, los cuales representan una alternativa como fuente de betalaínas y de antioxidantes.

Cuadro 2. Contenido (en mg equivalentes de ácido gálico/100 g de pulpa seca) de fenoles solubles totales (FST), ácidos fenólicos libres (AFL), glucosilados (AFG) y esterificados, en frutos de pitaya roja y naranja.

\begin{tabular}{lcccc}
\hline Muestra & FST & AFL & AFG & AFE \\
\hline Pitaya roja & $166.5 \mathrm{a} \pm 14.40 \mathrm{a}$ & $18.15 \mathrm{~b} \pm 0.04$ & $3.6 \mathrm{~b} \pm 0.31$ & $2.9 \mathrm{~b} \pm 0.23$ \\
Pitaya naranja & $52.8 \mathrm{~b} \pm 3.8$ & $28.35 \mathrm{a} \pm 5.67$ & $4.3 \mathrm{a} \pm 0.22$ & $5.6 \mathrm{a} \pm 0.66$ \\
\hline
\end{tabular}

Medias con letras iguales en una columna, no son estadísticamente diferentes (Tukey, 0.05). 

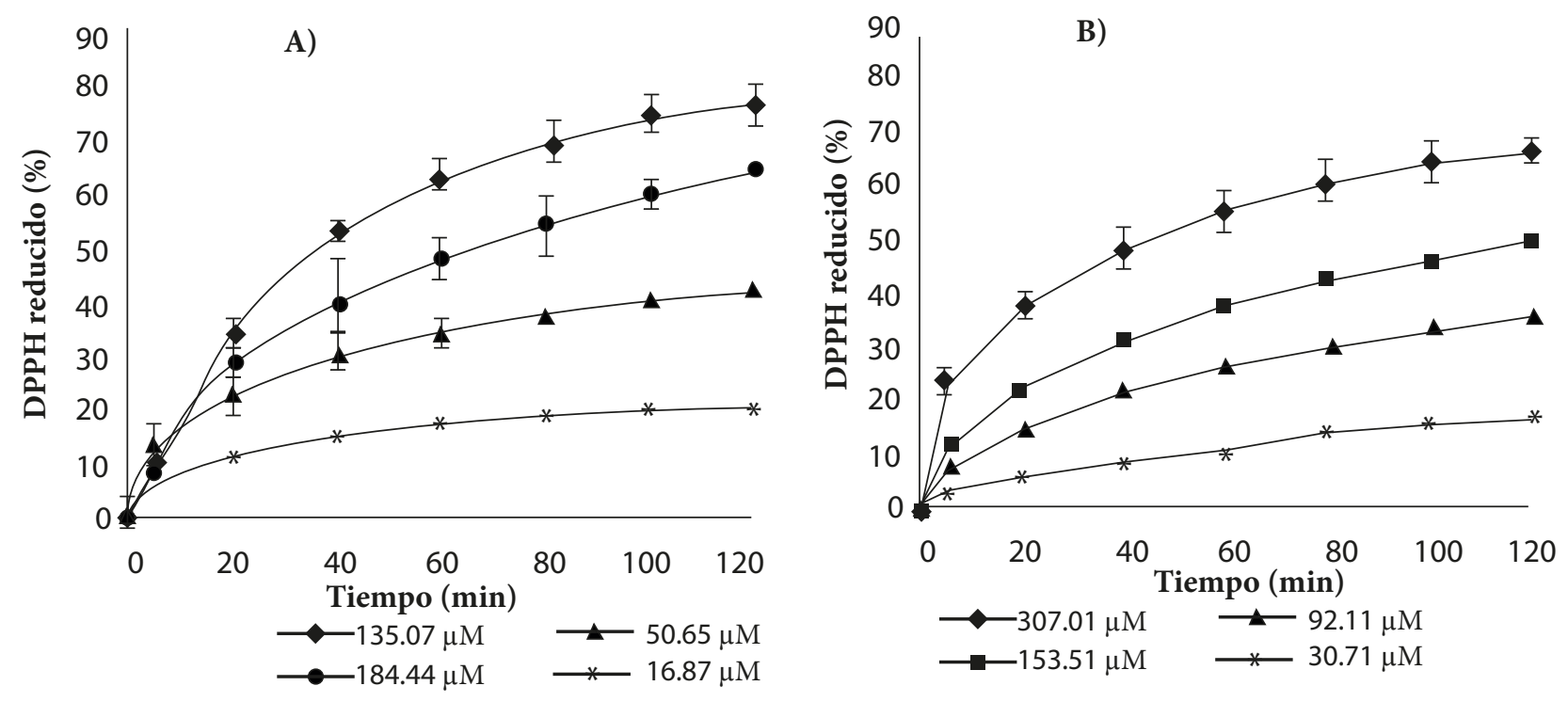

Figura 1. Porcentaje de DPPH reducido con cuatro concentraciones de los extractos metanólicos de betalaínas de Stenocereus griseus $\mathrm{H}$. (A, pitaya roja; B pitaya naranja).

\section{BIBLIOGRAFÍA}

Azeredo, H M C (2009) Betalains: properties, sources, applications, and stability- a review. Int. J. Food Sci. Tech. 44:2365-2376.

Bakan B, A C Bily, D Melcion, B Cahagnier, C Regnault-Roger, B J R Phylogene, D Richard-Molard (2003) Possible role of plant phenolics in the production of trichothecenes by Fussarium graminearum strains on different fracction of maize kernels. J. Agric. Food Chem. 5:2826-2331.

Cai Y, M Sun, H Corke (2003) Antioxidant activity of betalains from plants of the Amaranthaceae. J. Agric. Food Chem. 51: 2288-2294.

Castellanos-Santiago E, E M Yahia (2008) Identification and quantification of betalains from the fruits of 10 Mexican prickly pear cultivars by high-performance liquid chromatography and electrospray ionization mass spectrometry. J. Agric. Food Chem. 56: 5758-5764.

Cayupán C Y S, M J Ochoa, M A Nazareno (2011) Health-promoting substances and antioxidant properties of Opuntia sp. fruits. Changes in bioactive-compound contents during ripening process. Food Chem. 126:514-519.

Gandía-Herrero F, M Jiménez-Atiénzar, J Arbanes, F García-Carmona, J Escribano (2010) Stabilization of the bioactive pigment of Opuntia fruits througth maltodextrin encapsulation. J. Agric. Food Chem. 58:10646-10652.

Guzmán-Maldonado S H, G Herrera-Hernández, D Hernández-López, R Reynoso-Camacho, A Guzmán-Tovar, F Vaillant, P Brat (2010) Physicochemical, nutritional and functional characteristics of two underutilised fruit cactus species (Myrtillocactus) produced in central Mexico. Food Chem. 121:381-386.

Herbach K, C Maier, F C Stintzing, R Carle (2007) Effects of processing and storage on juice colour and betacyanin stability of purple pitaya (Hylocereus polyrhizus) juice. Eur. Food Res. Technol. 224:649658.

Kujala T S, J M Loponen, D K Klika, K Pihlaja (2000) Phenolics and betacyanins in red beetroot (Beta vulgaris) root: distribution an effect of cold storage on the content of total phenolics and three individual compounds. J. Agric. Food Chem. 48:5338-5372.

Luna-Morales C del C (2006) Clasificación y ordenación morfológica del fruto de variantes cultivadas de pitaya [Stenocereus pruinosus (Otto) Buxb.] en la Mixteca Baja, México. Rev. Chapingo S. Hort. 12:245-250.

Luna-Morales C del C, R Aguirre (2001) Variación morfológica del fruto y domesticación de Stenocereus pruinosus (OttoO Buxb. y S. stella- tus (Pfeiff.) Ricobb. (Cactaceae) en la Mixteca Baja, México. Rev. Fitotec. Mex. 24:213-221.

Liu Q, H Yao (2007) Antioxidant activities of barley seeds extracts. Food Chem. 102:732-737.

Osorio-Esquivel O, A Ortiz-Moreno, V B Alvarez, L Dorantes-Alvarez, M M Giusti (2011) Phenolics, betacyanins and antioxidant activity in Opuntia joconostle fruits. Food Res. Int. 44:2160-2168.

Peña-Varela G, Y Salinas-Moreno, R Ríos Sánchez (2006) Contenido de antocianinas totales y actividad antioxidante en frutos de frambuesa (Rubus idaeus L.) con diferente grado de maduración. Rev. Chapingo S. Hort. 2:159-163.

Robbins J R (2003) Phenolic acids in food: An overview of analytical methodology. J. Agric. Food Chem. 51: 2866-2887.

SAS Institute (2002) Statistical Analysis System Version 9.2. SAS Institute Inc., Cary, North Carolina, USA.

Shahidi F (2009) Nutraceuticals and functional foods: Whole versus processed foods. Trends Food Sci. Tech. 20:376-387.

Singleton V L, J J A Rossi (1965) Colorimetry of total phenolics with phosphomolybdic-phosphotungstic acid reagents. Amer. J. Enol. Vitic. 16:144-158.

Sreekantha D, M K Arunasree, R K Roy, C T Reddy, V G Reddy, P Reddanna (2007) Betanin a betacyanin pigment purified from fruits of Opuntia ficus-indica indices apoptosis in human chronic myeloid leukemia Cell line-k562. Phytomedicine14:739-746.

Stintzing F C, H M Herbach, M R Mosshammer (2005) Color, betalain pattern, and antioxidant properties of cactus pear (Opuntia spp.) clones. J. Agric. Food Chem. 53:442-451.

Strack D, T Vogt, W Schlieman (2003) Recent advances in betalain research. Phytochemistry 62:247-269.

Torres-Rodríguez A, Y Salinas-Moreno, S Valle-Guadarrama, I Alia-Tejacal (2011) Soluble phenols and antioxidant activity in mamey sapote (Pouteria sapota) fruits in postharvest. Food Res. Int. 44:1956-1961.

Villanueva-Tiburcio J E, L A Condenzo-Hoyos, E R Asquieri (2010) Antocianinas, ácido ascórbico, polifenoles totales y actividad antioxidante en la cáscara de camu-camu (Myrciaria dubi (H. B. K.) McVaugh). Ciëncia e Tecnol. Alim. 30:151-160.

Wu L-C, H Hsiu-Wen, C Yun-Chen, C Chih-Chung, L Yu-In, A H Ja-An (2006) Antioxidant and antiproliferative activities of red pitaya. Food Chem. 95:319-327.

Wybraniec S, Y Mizrahi (2002) Fruit flesh betacyanin pigments in Hylocereus cacti. J. Agric. Food Chem. 50:6086-6089. 